\title{
L'achondroplasie est associée à une mutation dans le domaine transmembranaire du récepteur 3 des facteurs de croissance fibroblastiques (FGFR3)
}

L'achondroplasie (ACH) est la forme la plus habituelle du nanisme. Sa fréquence est estimée à environ $1 \mathrm{p} 15000$. Elle est sporadique dans $80 \%$ des cas. Les formes héréditaires ont une transmission autosomique dominante avec une pénétrance de $100 \%$. Cliniquement, c'est un nanisme rhizomélique (proximal) avec macrocéphalie relative, hyperlordose lombaire, mortalité accrue chez l'enfant et l'adulte mais sans retard intellectuel. Le tableau est beaucoup plus sévère chez les homozygotes qui meurent en général très tôt. Le gène de l'ACH a été localisé sur la partie distale du bras court du chromosome 4 , et des travaux récents de plusieurs équipes, dont une française, l'ont fixé avec précision en $4 \mathrm{pl} 6$ $\left(\mathrm{m} / \mathrm{s}, n^{\circ} 4\right.$, vol. 10, p. 486, [1]). Cette région, voisine de celle du gène de la maladie de Huntington, a été pour cette raison l'objet de recherches intenses ; on y a découvert notamment, outre celui de la maladie de Huntington, les gènes de la maladie de Hurler-Scheie ( $\alpha$ iduronidase) et d'une forme de cécité nocturne par anomalie de la sous-unité $\beta$ de la phosphodiestérase du GMPc. En 1991 [2], a été caractérisé dans cette région un gène, momentanément considéré comme un candidat pour la maladie de Huntington, et qui s'est révélé être un récepteur de facteur de croissance, FGFGR3.

Les travaux de deux équipes, l'une américaine [3] et l'autre française [4], viennent de démontrer que FGFR3 est en fait le gène respon- sable de l'achondroplasie et que des mutations récurrentes d'un codon précis du domaine transmembranaire de FGFR3 rendent compte de tous les cas étudiés à ce jour.

On sait que les facteurs de croissance fibroblastiques forment un groupe nombreux (au moins neuf membres) et qu'ils agissent par l'intermédiaire de récepteurs de surface cellulaire à activité tyrosine kinase. On connaît actuellement quatre récepteurs différents, FGFR1 à 4 ; ils se ressemblent par leur structure et leur mode de fonctionnement, mais ont des profils d'expression très différents au cours du développement. Surtout, la distribution du FGFR3 est beaucoup plus limitée que celle des récepteurs 1 et 2 : outre le système nerveux, c'est avant tout dans le cartilage préosseux qu'on le trouve. Ces caractères (localisation chromosomique, rôle dans l'ossification), ont incité à explorer le FGFR3 chez des malades atteints d'ACH. La séquence du FGFR3 est connue depuis 1991 [2, 6]. La protéine compte 806 acides aminés mais l'organisation du gène humain n'est pas encore connue en détail. La structure générale de la protéine comporte, après une séquence signal, trois domaines extracellulaires du type immunoglobuline, une région unique transmembranaire, puis deux domaines tyrosine kinase (figure 1).

La méthode d'analyse mise en œuvre par Shiang et al. [3,5] fut la DGGE (denaturing gradient gel electrophoresis) effectuée sur l'ARN de lymphoblastes ou fibroblastes après transcription inverse et amplification. Sur quinze des seize malades examinés, on retrouva une même anomalie, une transition $\mathrm{G} \rightarrow \mathrm{A}$ au nucléotide 1138 entraînant un changement Gly $\rightarrow$ Arg au codon 380 de la protéine mure. Le cas $n^{\circ} 16$ présentait, sur le même nucléotide, une transversion $G \rightarrow F$, provoquant le même changement d'acide aminé. Pour tenter de simplifier le criblage, on passa à l'ADN génomique, car la mutation usuelle crée un site pour l'enzyme Sfc 1. On retrouva les résultats obtenus avec l'ADNc, confirmant que tous les malades analysés jusqu'à présent portent une mutation au nucléotide 1138, qu'il s'agisse de formes familiales ou de cas sporadiques. Chez trois de ces derniers, on a pu vérifier que les parents étaient indemnes, et que les mutations s'étaient produites de novo. Enfin, parmi les sujets étudiés, figurent des homozygotes qui portent la même mutation sur les deux allèles.

La méthode d'analyse utilisée par l'équipe française $[4,5]$ est différente (SSCP, single strand conformation polymorphism) mais les conclusions de Rousseau et al. sont identiques. En effet, 23/23 achondroplases ( 17 cas sporadiques, 6 formes familiales) sont porteurs de la mutation Gly $\rightarrow$ Arg au codon 380 dans le domaine transmembranaire de FGFR3 et cette mutation ségrège avec la maladie dans les formes héritées. De plus, dans la série française, la mutation $G \rightarrow$ A est retrouvée chez 22/23 patients, la transversion 


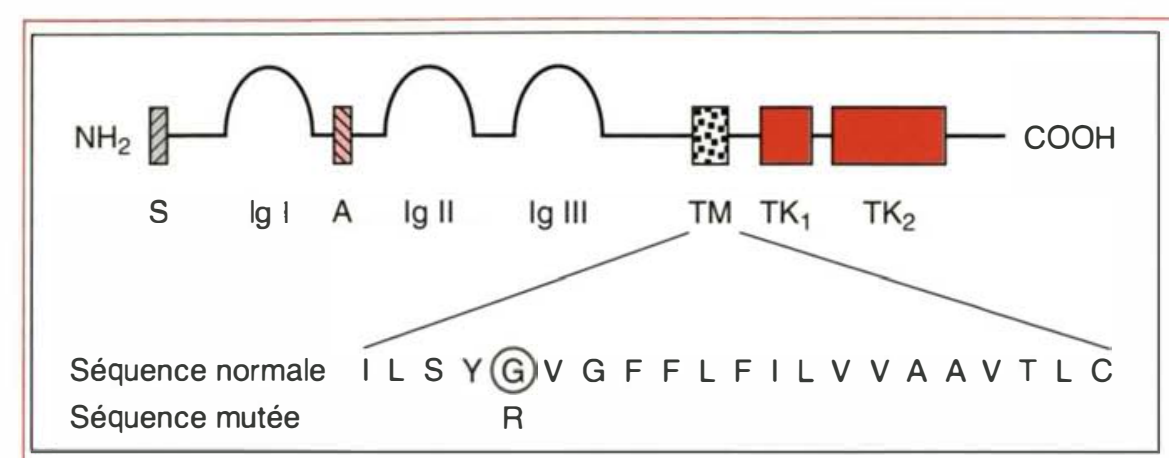

Figure 1. Structure du FGFR3 et site de la mutation. De l'extrémité $\mathrm{NH}_{2}$ à l'extrémité $\mathrm{COOH}$ : peptide signal $S$; les trois domaines immunoglobuline $1 \mathrm{~g}$, avec entre 1 et 2 , une région acide $A$; la zone transmembranaire $T M$; les deux domaines tyrosine kinase TK. La zone entourant la mutation est détaillée. (D'après [4].)

$G \rightarrow C$ n'étant observée qu'une seule fois.

Les résultats obtenus par Shiang et al. [3] et Rousseau et al. [4] sont intéressants à plusieurs titres. Il est encore difficile de comprendre par quel mécanisme une anomalie d'un récepteur aboutit à un tableau clinique aussi particulier. Il n'y a, en tout cas, pas de vicariance par d'autres récepteurs. Pour interpréter les résultats, il faut d'abord remarquer que dans le syndrome de Wolf-Hirschhorn, qui est dû à une délétion terminale du $4 \mathrm{p}\left(\mathrm{m} / \mathrm{s} n^{\circ} 4\right.$, vol. 8, p. 395), la délétion englobe le gène du FGFR3 et, cependant, on n'y observe pas les signes de l'ACH. La perte d'un allèle ne suffit donc pas à produire ce syndrome dominant. On doit donc supposer que, dans l'ACH, une anomalie de fonction entraîne l'impossibilité de s'engager dans des dimères fonctionnels avec d'autres sous-unités de FGFR3 ou d'autres récepteurs. La mutation qui siège sur le segment transmembranaire modifie, sans doute, la structure de la molécule. Reste une question : pourquoi l'atteinte du même nucléotide, dans tous les cas étudiés, familiaux ou sporadiques ? Il peut certes y avoir un point chaud de mutation, d'autant qu'il s'agit d'un îlot CpG. Mais on est en droit de penser que, si une mutation se produit dans un autre domaine, que ce soit dans les domaines immunoglobulines ou tyrosine kinase, d'autres conséquences s'ensuivront. Elles pourraient être d'autant plus diverses que le FGFR3 possède des variants d'épissage et peut se combiner à nombre d'autres récepteurs, ainsi qu'à plusieurs ligands FGF [7]. En attendant, il semble qu'il existe une spécificité étroite, dans le segment transmembranaire, et peut-être même dans le seul codon 380 , qui provoque une $\mathrm{ACH}$. On peut risquer une comparaison avec les hémoglobinoses : parmi des centaines de mutations possibles, une seule entraîne une drépanocytose. Enfin, sur le plan pratique, on peut proposer un diagnostic prénatal si (et seulement si) les parents sont tous deux hétérozygotes. Il est justifié à la fois par le caractère létal de la forme homozygote, et par les difficultés de la grossesse chez les femmes atteintes d'ACH.

\section{Jean-Claude Dreyfus Francis Rousseau Jacky Bonaventure Martine Le Merrer Arnold Munnich}

1. Le Merrer M, Rousseau F, Legeai-Mallet l. A gene for achondroplasia-hypochondroplasia maps to chromosome 4p. Nature Genet $1994 ; 6$ : 318-21.

2. Thompson LM, Plummer S, Schalling M, Altherr MR, Gusella JF, Housman DE, Wasmuth.JJ. A gene encoding a fibroblast growth factor receptor isolated from the Huntington disease gene region of human chromosome 4 . Genomics 1991 ; 11 : 1133-42.

3. Shiang $\mathrm{R}$, Thompson LM, Zhu YZ, Church DM, Fielder TJ, Bocian M, Winokur ST, Wasmuth .JJ. Mutations in the transmembrane domain of FGFR3 cause the most common genetic form of dwarfism, achondroplasia. Cell $1994 ; 78: 335-42$.

4. Rousseau F, Bonaventure J, Legeai-Mallet L, Pelet A, Rozet JM, Maroteaux P, Le Merrer M, Munnich A. Mutations of the fibroblast growth factor receptor 3 gene in achondroplasia. Nature 1994 (sous presse).

5. Dreyfus JC, Akli S, Poenaru L Maladies de Tay. Sachs et de Sandhoff: les déficits en $\beta$ hexosominidases : modèles de maladies des lysosomes. médecine/sciences $1992 ; 8: 797-804$.

6. Keegan K, Johnson DE, Williams LT, Hayman $\mathrm{MJ}$. Isolation of an additional member of the fibroblast growth factor receptor family, FGFR3. Proc Natl Acad Sci USA 1991 ; 88 : 1095-9.

7. Chellaiah AT, McEwen DG, Werner S, Xu J, Ornitz DM. Fibroblast growth factor receptor (FGFR) 3. J Biol Chem 1994 ; 269 : 11620-7.
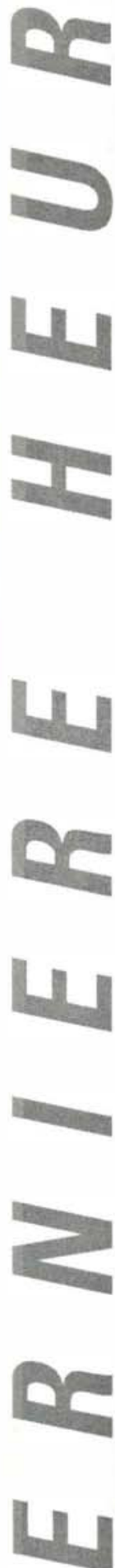